\title{
Universal crossing probability in anisotropic systems
}

\author{
L. TURBAN \\ Laboratoire de Physique des Matériaux, UMR CNRS 7556, Université Henri Poincaré \\ (Nancy 1) - BP 239, 54506 Vandouvre lès Nancy Cedex, France \\ PACS. 64.60.Ak - Renormalization-group, fractal, and percolation studies of phase transi- \\ tions. \\ PACS. 05.50.+q - Lattice theory and statistics (Ising, Potts, etc.). \\ PACS. 02.50.-r - Probability theory, stochastic processes, and statistics.
}

\begin{abstract}
Scale-invariant universal crossing probabilities are studied for critical anisotropic systems in two dimensions. For weakly anisotropic standard percolation in a rectangularshaped system, Cardy's exact formula is generalized using a length-rescaling procedure. For strongly anisotropic systems in $1+1$ dimensions, exact results are obtained for the random walk with absorbing boundary conditions, which can be considered as a linearized mean-field approximation for directed percolation. The bond and site directed percolation problem is itself studied numerically via Monte Carlo simulations on the diagonal square lattice with either free or periodic boundary conditions. A scale-invariant critical crossing probability is still obtained, which is a universal function of the effective aspect ratio $r_{\text {eff }}=c r$ where $r=L / t^{z}, z$ is the dynamical exponent and $c$ is a non-universal amplitude.
\end{abstract}

Introduction. - The study of universal crossing probabilities in the standard random percolation problem at criticality has been the subject of much interest over the last decade [16] (see ref. [7] for a review). At the percolation threshold, $p_{\mathrm{c}}$, on a rectangular lattice with length $L_{\|}$and width $L_{\perp}$, the probability to have at least one cluster crossing the system in one of the two directions (or in both) is a scale-invariant quantity in the continuum limit where the lattice spacing $a \rightarrow 0$. Actually, the crossing probability is a function $\pi(r)$ of the aspect ratio, $r=L_{\perp} / L_{\|}$, and depends also on the boundary conditions. It remains universal in the sense that it does not depend on the lattice type or whether one is considering site, bond or site-bond percolation.

Following the pioneering numerical work of Langlands et al. [1], Cardy [2] obtained an analytical expression for the crossing probability between opposite sides of a rectangle. He combined the correspondence between percolation and the limit $q \rightarrow 1$ of the $q$-state Potts model [8] with boundary conformal field theory techniques to calculate the crossing probability between two segments on the boundary of a half-plane at criticality. A conformal mapping could then be used to transform the half-plane result into the rectangular geometry. Cardy's and another crossing probability formula have been recently proven rigorously [4]. Monte Carlo [5] and exact results [6] have been obtained for the probability to find $n$ incipient spanning clusters connecting two disjoint segments at the boundary of a finite system. More

(C) EDP Sciences 
recently, crossing probabilities on same-spin Ising clusters in two dimensions have been also considered [9].

Let us notice that the existence of a scale-invariant crossing probability is not a trivial result. In the case of ordinary percolation, for example, it is linked to the vanishing, in the limit $q \rightarrow 1$, of the scaling dimension $x(q)$ of a boundary condition changing operator of the Potts model [2].

In this letter, we investigate the behaviour of crossing probabilities in anisotropic critical systems. We first give an exact treatment of weakly anisotropic standard percolation. We show how a length rescaling technique, which has been used in other contexts [10], allows to generalize Cardy's exact results in this situation. Then we turn to crossing probabilities in strongly anisotropic systems. To our knowledge, such problems have not been considered before, at least with the appropriate anisotropic scaling analysis. We start with the random walk with absorbing boundary conditions in the continuum limit which can be considered as a linearized mean-field approximation [11] for directed percolation [12 14] and illustrates on an exactly solvable example the behaviour of the crossing probability in strongly anisotropic systems. Finally we present the results of Monte Carlo simulations for the crossing probability in directed percolation, a strongly anisotropic problem which has so far defied all attempts at an exact solution, even in two dimensions.

Weakly anisotropic percolation. - We consider the random bond percolation on a square lattice with anisotropic bond occupation probabilities. The system is rectangular and the bond probability is $p_{\|}\left(p_{\perp}\right)$ in the direction parallel to the side with length $L_{\|}\left(L_{\perp}\right)$. The correlation lengths, $\xi_{\|}=\hat{\xi}_{\|} t^{-\nu}, \xi_{\perp}=\hat{\xi}_{\perp} t^{-\nu}$, diverge along the critical line where $t=0$ with the same exponent $\nu$ for both directions but different amplitudes $\hat{\xi}_{\|}$and $\hat{\xi}_{\perp}$. The isotropy can be restored [10] by rescaling the lattice parameter anisotropically so that $\xi_{\|} a_{\|}=\xi_{\perp} a_{\perp}$. The aspect ratio $r$ is then changed into an effective one

$$
r_{\mathrm{eff}}=\frac{L_{\perp} a_{\perp}}{L_{\|} a_{\|}}=\frac{L_{\perp} / \xi_{\perp}}{L_{\|} / \xi_{\|}}=\frac{\hat{\xi}_{\|}}{\hat{\xi}_{\perp}} r
$$

In the second expression the effective aspect ratio involves the lengths measured in units of the corresponding correlation lengths.

The critical amplitude ratio $c=\hat{\xi}_{\|} / \hat{\xi}_{\perp}$, which is non-universal, is known for the $q$-state Potts model on the square lattice [15]. In the percolation limit, $q \rightarrow 1$, one obtains:

$$
c=\tan \frac{3 u}{2}, \quad \frac{\sin u}{\sin (\pi / 3-u)}=\frac{p_{\mathrm{c} \|}}{1-p_{\mathrm{c} \|}}=\frac{1-p_{\mathrm{c} \perp}}{p_{\mathrm{c} \perp}}
$$

which solves the problem for a weak anisotropy. When the anisotropy axis is not parallel to one edge of the rectangle, the rescaling leads to an isotropic system with the shape of a parallelogram.

Random walk. - Standard conformal methods do not apply in strongly anisotropic systems. The simplest example of such a system is the random walk in $1+1$ dimensions, which may be considered as a directed walk in space-time. In the continuum limit, the probability density $P(x, t)$ satisfies the diffusion equation

$$
\frac{\partial P}{\partial t}=D \frac{\partial^{2} P}{\partial x^{2}}
$$




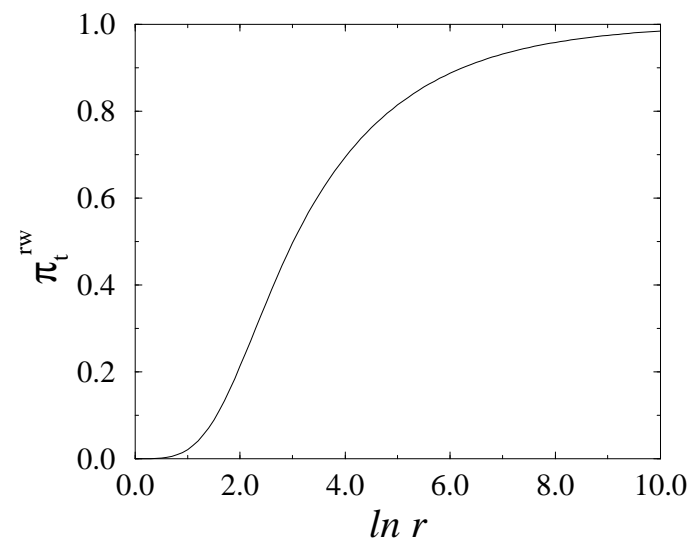

Fig. 1 - Crossing probability in the time direction for a random walk with absorbing boundary conditions at $l=0$ and $L$ and a uniform initial probability density at $t=0 . \quad r=L^{2} / t$ is the scale-invariant aspect ratio of the system and $D=1$.

We assume a uniform initial probability density $P(x, 0)=1 / L$ and absorbing boundary conditions at $x=0$ and $L$. The solution is then obtained as the following eigenvalue expansion:

$$
P(x, t)=\sum_{p=0}^{\infty} \frac{4}{(2 p+1) \pi L} \sin \left[(2 p+1) \frac{\pi x}{L}\right] \exp \left[-\frac{(2 p+1)^{2} \pi^{2} D t}{L^{2}}\right] .
$$

On the rectangle $L \times t$, the crossing probability in the time direction is given by:

$$
\pi_{t}^{\mathrm{rw}}=\int_{0}^{L} P(x, t) \mathrm{d} x=\sum_{p=0}^{\infty} \frac{8}{(2 p+1)^{2} \pi^{2}} \exp \left[-\frac{(2 p+1)^{2} \pi^{2} D t}{L^{2}}\right] .
$$

Thus the crossing probability shown in fig. 1 is a universal function of the scaled variable $c L^{z} / t$, product of the aspect ratio $r=L^{z} / t$, where $z=2$ is the dynamical exponent for the random walk, by the non-universal inverse diffusion constant $c=D^{-1}$.

In strongly anisotropic systems, such a behaviour generally follows from anisotropic scaling 16. Under the anisotropic scale transformation, $L^{\prime}=L / b, t^{\prime}=t / b^{z}$, the crossing probability behaves as:

$$
\pi_{t}(L, t)=b^{-x_{\pi_{t}}} \pi_{t}\left(\frac{L}{b}, \frac{t}{b^{z}}\right) .
$$

For a dimensionless crossing probability, $x_{\pi_{t}}=0$, one obtains the scale-invariant behaviour mentioned above with $b=L$.

The probability density for a walker starting at $t=0$ with uniform probability on the segment $[0, L]$ to die at time $t$ is given by

$$
-\frac{\mathrm{d} \pi_{t}^{\mathrm{rw}}}{\mathrm{d} t}=\frac{8 D}{L^{2}} \sum_{p=0}^{\infty} \exp \left[-\frac{(2 p+1)^{2} \pi^{2} D t}{L^{2}}\right]=\frac{4 D}{L^{2}} \vartheta_{2}\left[\exp \left(-\frac{4 \pi^{2} D t}{L^{2}}\right)\right],
$$

where $\vartheta_{2}(u)=\sum_{p=-\infty}^{+\infty} u^{(p+1 / 2)^{2}}$ is a Jacobi theta function.

Since the linearized mean-field approximation for directed percolation [11, 13] is also governed by the diffusion equation (3), the mean-field crossing probability for directed percolation is given by (5) when the aspect ratio is sufficiently small. 

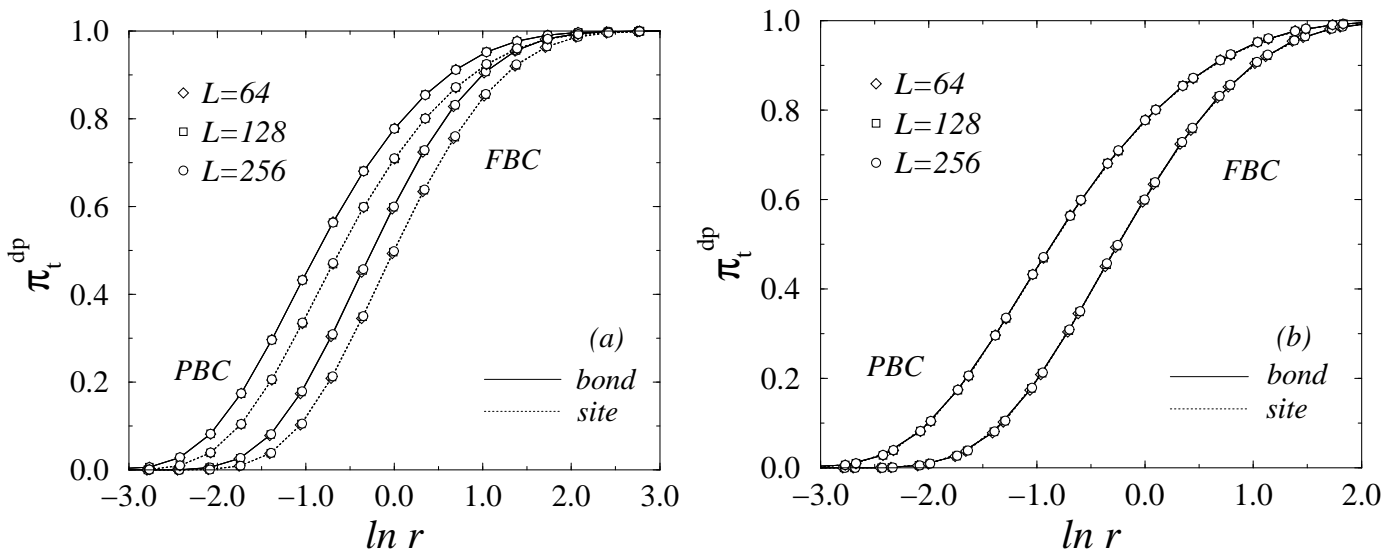

Fig. 2 - (a) Crossing probability $\pi_{t}^{\mathrm{dp}}$ in the time direction as a function of the logarithm of the aspect ratio $r=L^{z} / t$ for site and bond directed percolation on the diagonal square lattice with either periodic or free boundary conditions in the space direction. The $L$ sites are wet at $t=0$. (b) Shifting the curves for the site problems by the same amount, the data collapse on a single scale-invariant universal curve for each type of boundary conditions.

Directed percolation. - The critical crossing probability in the time direction, $\pi_{t}^{\mathrm{dp}}$, has been studied via Monte Carlo simulations for bond and site directed percolation on the square lattice, with the time axis along the diagonal direction. Space and time coordinates take integer and half-integer values alternatively on successive spatial rows and $t \geq 0$. There are two directed bonds leaving a site at $(x, t)$ and terminating on the sites at $(x \pm 1 / 2, t+1 / 2)$.

Sites may be wet or dry and a directed percolation cluster is a collection of connected wet sites starting from some source at $t=0$ 12 14. In the bond problem, bonds are open with probability $p$. A site is wet at time $t+1 / 2$ when it is connected through an open bond to a site which was wet at time $t$. In the site problem, sites are occupied with probability $p$ and all the bonds are open. A site is wet at time $t+1 / 2$ when it is occupied and connected to a site which was wet at time $t$. A scale-invariant crossing probability is obtained when all the sites are wet in the initial state at $t=0$.

The critical crossing probability was calculated for $10^{6}$ samples with sizes $L=2^{3}$ to $2^{8}$ and different values of the aspect ratio, $r=L^{z} / t$, ranging from $r \simeq 2^{-5}$ to $2^{5}$. The percolation thresholds $p_{\mathrm{c}}^{\text {bond }}=0.644700185(5), p_{\mathrm{c}}^{\text {site }}=0.70548522(4)$ and the dynamical exponent $z=$ $1.580745(10)$ were taken from ref. 17]. Either periodic boundary conditions (PBC) or free boundary conditions (FBC) were used at $l=1$ and $L$. The samples were grouped into 20 packets in order to evaluate the errors on $\pi_{t}^{\mathrm{dp}}$. The raw data $\pi_{t}^{\mathrm{dp}}$ versus $\ln r$ are shown in fig. $2 \mathrm{a}$ for the largest sizes. The statistical errors are actually smaller than the size of the symbols. The scale invariance is manifest and finite size corrections to the leading behaviour are quite weak. There is a shift in $\ln r$ between the curves for site and bond percolation which is the same for PBC and FBC. As shown in fig. 2b, an universal curve is obtained for each type of boundary condition after the curves corresponding to the site problem have been shifted by the same amount.

In this strongly anisotropic problem, the correlation lengths, $\xi_{\perp}$ in the space direction and 


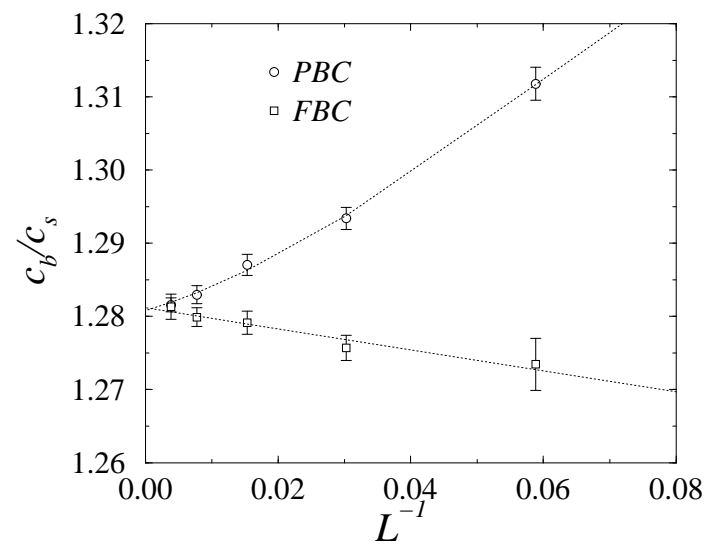

Fig. 3 - Non-universal amplitude ratio $c_{\mathrm{b}} / c_{\mathrm{s}}$ as defined in eq. (9) as a function of the inverse of the system size $L$. The dotted line corresponds to a cubic (quadratic) fit of the data for periodic (free) boundary conditions.

$\xi_{\|}$in the time direction, diverge as

$$
\xi_{\perp}=\hat{\xi}_{\perp}\left|p-p_{\mathrm{c}}\right|^{-\nu}, \quad \xi_{\|}=\hat{\xi}_{\|}\left|p-p_{\mathrm{c}}\right|^{-z \nu} .
$$

Thus, generalizing the behaviour for the weakly anisotropic system in eq. (1), for each type of boundary conditions, $i=F B C$ or $P B C$, one expects the crossing probability $\pi_{t}^{\mathrm{dp}}$ to be an universal function $f_{i}\left(r_{\text {eff }}\right)$ of the scale-invariant ratio

$$
r_{\mathrm{eff}}=\frac{\left(L / \xi_{\perp}\right)^{z}}{t / \xi_{\|}}=c r, \quad c=\frac{\hat{\xi}_{\|}}{\left(\hat{\xi}_{\perp}\right)^{z}},
$$

in agreement with anisotropic scaling in eq. (6). As above for weakly anisotropic percolation and for the random-walk, the constant $c$ is non-universal and takes different values, $c_{\mathrm{s}}$ and $c_{\mathrm{b}}$, for the site and bond directed percolation on the diagonal square lattice.

For a given type of boundary conditions, the crossing probability $\pi_{t}^{\mathrm{dp}}$ is the same for couples of values of the aspect ratio $r_{\mathrm{s}}$ and $r_{\mathrm{b}}$ such that the arguments of the universal function $f_{i}\left(r_{\mathrm{eff}}\right)$ are the same for the site and bond problems. This happens when $c_{\mathrm{s}} r_{\mathrm{s}}=c_{\mathrm{b}} r_{\mathrm{b}}$. Thus, with a logarithmic scale, the two curves have a relative shift

$$
\ln r_{\mathrm{s}}-\ln r_{\mathrm{b}}=\ln \left(\frac{c_{\mathrm{b}}}{c_{\mathrm{s}}}\right) .
$$

This shift was estimated for the different sizes for 6 points in the ascending part of the the curves. Since the points for the site and bond problems correspond to slightly different ordinates, a direct estimation of the shift was not possible. Instead we used a polynomial interpolation for the curve corresponding to the bond problem and the value of the abscissa associated with the common value of the ordinate was determined through the tangent method. The results for the ratio of non-universal amplitudes as a function of the inverse size are shown in fig. 3 for PBC and FBC. They converge from above and from below to the common asymptotic value

$$
\frac{c_{\mathrm{b}}}{c_{\mathrm{s}}}=1.281(1)
$$


EUROPHYSICS LETTERS

corresponding to the shift $\ln r_{\mathrm{s}}-\ln r_{\mathrm{b}}=0.2476(8)$ which was used to obtain the data collapse in fig. $2 b$.

Final remarks. - The same universal crossing probability is obtained for any finite homogeneous density of wet sites in the initial state at $t=0$ although the finite-size corrections to the leading scale-invariant behaviour is stronger at large values of $r$ (small $t$ ) when the initial density decreases. With a single wet site at $l=L / 2$ in the initial state, i.e., for an asymptotically vanishing initial density, the crossing probability remains a function of $r_{\text {eff }}$ but the scale-invariance is lost, $\pi_{t}^{\mathrm{dp}}$ decaying as $L^{-\beta / \nu}$. The probability $\pi_{t}^{\mathrm{dp}}(n)$ to find $n$ clusters crossing the system in the time direction at $p_{\mathrm{c}}$ has been also studied. Details will be given elsewhere.

This work can be extended by considering the crossing probability on same-spin clusters in strongly anisotropic spin systems, like the three-dimensional uniaxial ANNNI model at the Lifshitz point, for which presumably exact results for the form of the correlation functions, following from a generalization of local scale invariance [18], have been obtained recently [19].

The author wishes to thank LEv SHCHUR for a stimulating introductory talk about spanning clusters.

\section{REFERENCES}

[1] Langlands R. P., Pichet C., Pouliot P. and Saint-Aubin Y., J. Stat. Phys., 67 (1992) 553.

[2] Cardy J. L., J. Phys. A, 25 (1992) L201.

[3] Langlands R. P., Pouliot P. and Saint-Aubin Y., Bull. AMS, 30 (1994) 1; Pinson T. H., J. Stat. Phys., 75 (1994) 1167; Saint-Aubin Y., Physica A, 221 (1995) 41; Ziff R. M., J. Phys. A, 28 (1995) 1249; Ziff R. M., J. Phys. A, 28 (1995) 6479; Watts G. M. T., J. Phys. A, 29 (1996) L363; Hu C.-K. and Lin C.-Y., Phys. Rev. Lett., 77 (1996) 8; Hovi J.-P. and Aharony A., Phys. Rev. E, 53 (1996) 235; Ziff R. M., Lorenz C. D. and Kleban P., Physica A, 266 (1999) 17; Kleban P., Physica A, 281 (2000) 242;

[4] Smirnov S., C. R. Acad. Sci. Paris, Série I, 333 (2001) 239; Schramm O., Elect. Comm. Probab., 6 (2001) 115.

[5] Sen P., Int. J. Mod. Phys. C, 7 (1996) 603; ibid, 8 (1997) 229; Shchur L. N. and Kosyakov S. S., Int. J. Mod. Phys. C, 8 (1997) 473; Nucl. Phys. B (Proc. Suppl.), 63A-C (1998) 664; Grassberger P. and Nadler W., cond-mat/0010265; Grassberger P., cond-mat/0201313.

[6] Aizenman M., Nucl. Phys. B, 485 (1997) 551; Aizenman M., Duplantier B. and Aharony A., Phys. Rev. Lett., 83 (1999) 1359; Cardy J. L., J. Phys. A, 31 (1998) L105.

[7] Cardy J. L., Lectures on Conformal Invariance and Percolation, Bunkyo-ku, Tokyo (2001), math-ph/0103018.

[8] Kasteleyn P. W. and Fortuin C. M., J. Phys. Soc. Japan (Suppl.), 26 (1969) 11.

[9] Langlands R. P., Lewis M.-A. and Saint-Aubin Y., J. Stat. Phys., 98 (2000) 131; Lapalme E. and Saint-Aubin Y., J. Phys. A, 34 (2001) 1825; Arguin L.-P. and Saint-Aubin Y., hep-th/0109138.

[10] Barber M. N., Peschel I. and Pearce P. A., J. Stat. Phys., 37 (1984) 497.

[11] Kaiser C. and Turban L., J. Phys. A, 28 (1995) 351.

[12] Broadbent S. R. and Hammersley J. M., Proc. Camb. Philos. Soc., 53 (1957) 629.

[13] Kinzel W., Percolation Structures and Processes, Annals of the Israel Physical Society, edited by G. Deutscher, R. Zallen and J. Adler, Vol. 5 (Israel Physical Society, Haifa) 1983, pp. 425-445.

[14] Hinrichsen H., Adv. Phys., 49 (2000) 815. 
[15] Kim D. and Pearce P. A., J. Phys. A, 20 (1987) L451.

[16] Binder K. and Wang J.-S., J. Stat. Phys., 55 (1989) 87. See also Hucht A., condmat/0202142.

[17] Jensen I., J. Phys. A, 32 (1999) 5233.

[18] Henkel M., hep-th/0205256.

[19] Pleimling M. and Henkel M., Phys. Rev. Lett., 87 (2001) 125702. 